2. To: (Receiving organization)

Distribution

5. Proj./Prog./Dept./Div.:

SNF Project

8. Originator Remarks:

For Release

11. Receiver Remarks:
3. From: (originating organization) Process Engineering

6. Design Authority/ Design Agent/Cog. Engr.:

M. J. Packer

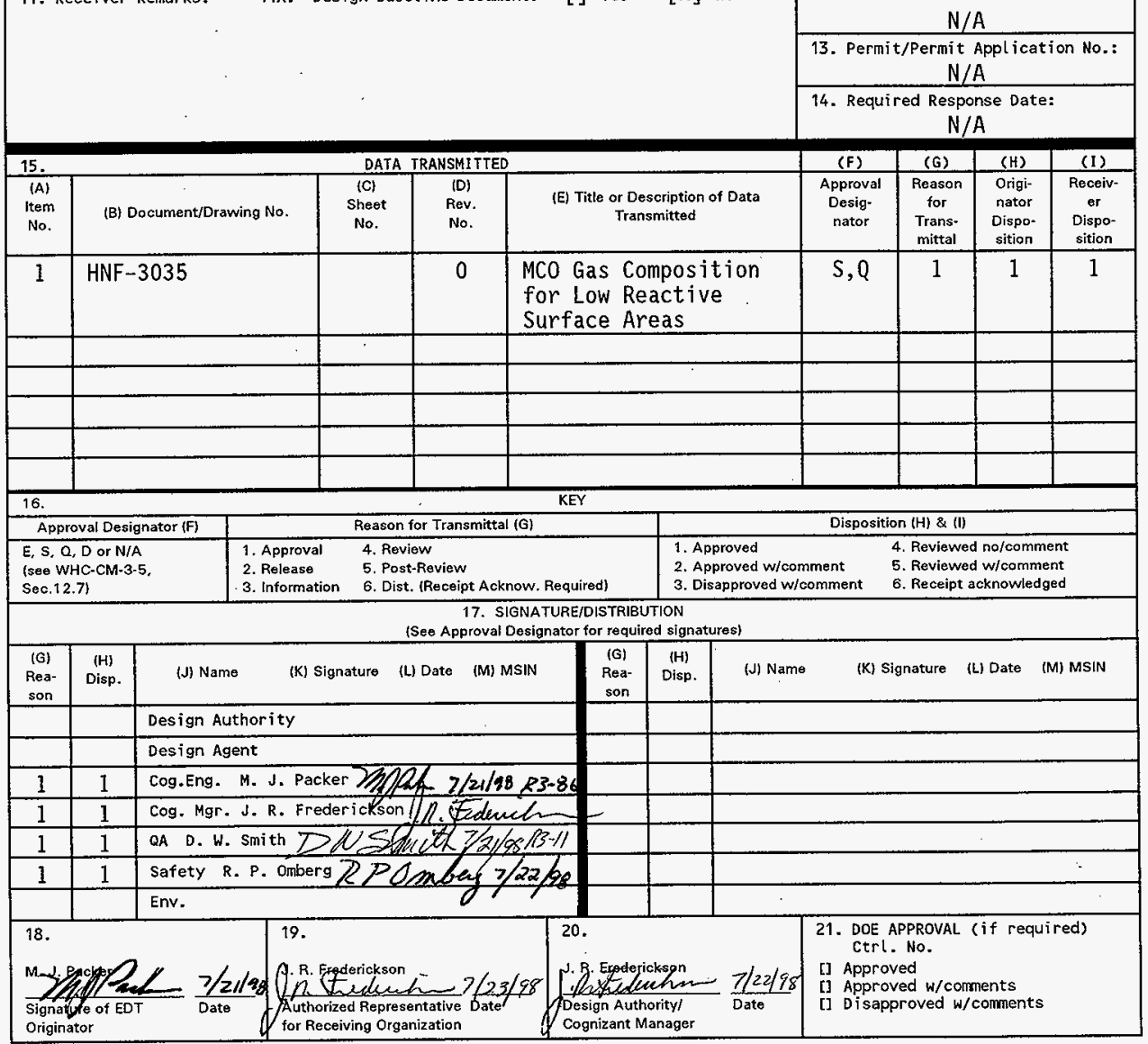

BD - 7400-172-2 (05/96) GEF097 
HNF- 3035, Rev. 0

\title{
MCO GAS COMPOSITION FOR LOW REACTIVE SURFACE AREAS
}

\author{
M. J. Packer
}

DE\&S Hanford Inc.

Richland, WA 99352

U.S. Department of Energy Contract DE-AC06-96RL13200

EDT/ECN: 625223

UC: 510

Org Code: 2 T950

Charge Code: LB051

B\&R Code: EW7040000 Total Pages: 20

Key Words: Reactive Surface Area, Backfill Overpressure, MCO

Abstract: This calculation adjusts modelled output (HNF-SD-SNF-TI-040, Rev. 2) by considering lower reactive fuel surface areas and by increasing the input helium backfill overpressure from 0.5 to $1.5 \mathrm{~atm}$ ( $2.5 \mathrm{~atm}$ abs) to verify that MCO gas-phase oxygen concentrations can remain below 4 mole \% over a 40 year interim period under a 'worst case' condition of zero reactive surface area. Added backfill gas will dilute any gases generated during interim storage and is a strategy within the current design capability. The zero reactive surface area represents a hypothetical 'worst case' example where there is no fuel scrap and/or damaged spent fuel rods in an MCO. Also included is a hypothetical case where only $\mathrm{K}$ East fuel exists (no $\mathrm{Al}(\mathrm{OH})_{3}$ ) in an $\mathrm{MCO}$ with an added backfill overpressure of $0.5 \mathrm{~atm}(1.5 \mathrm{~atm}$ abs).

MATHCAD is a trademark of Mathsoft.

TRADEMARK DISCLAIMER. Reference herein to any specific commercial product, process, or sexvice by trade name, trademark, manufacturer, or otherwise, does not necessarily constitute or imply its endorsement, recommendation, or favoring by the United states Government or any agency thereof or its contractors or subcontractors.

Printed in the united states of America. To obtain copies of this document, contact: Document Control Services, P.0. Box 950, Mailstop H6-09 $372-2420 ; \operatorname{Fax}(509) 376-4989$.
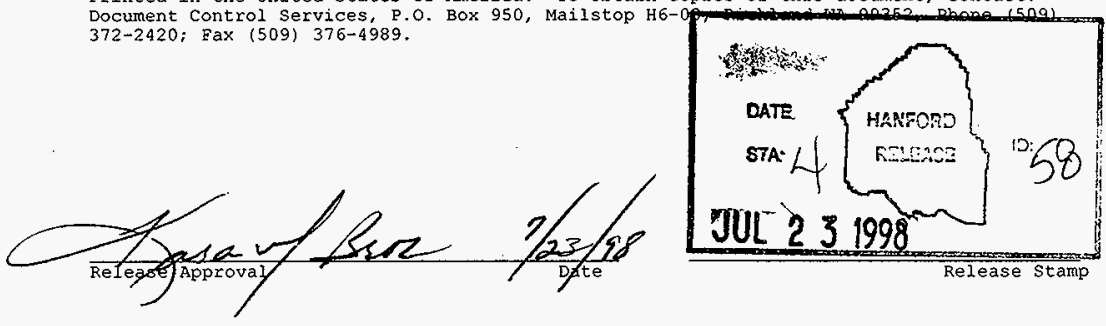

Approved for Public Release 


\section{REVIEW CHECKLIST}

Document Reviewed:

"MCO Gas Composition for Low Reactive Surface Areas," HNF-3035, Rev. 0, M. J. Packer

Scope of Review:

Assumptions, Methodology, Conclusions

Yes No NA

\ $\square \square$

* Previous reviews complete and cover analysis, up to scope of this review, with no gaps. Problem completely defined.

Accident scenarios developed in a clear and logical manner.

$\begin{array}{lll}x & \square & \square \\ \square & \square & \square\end{array}$

Necessary assumptions explicitly stated and supported.

冈 $\square \square$ Computer codes and data files documented.

(x) $\square \square$

Data used in calculations explicitly stated in document.

$x \square \square$

Data checked for consistency with original source information as applicable.

x $\square \square$

Mathematical derivation checked including dimensional consistency of results.

$\mathrm{x}$

Models appropriate and used within range of validity or use outside range of established validity justified.

Hand calculations checked for errors. Spreadsheet results should be treated exactly the same as hand calculations.

[ $\square \square$ Software input correct and consistent with document reviewed.

四 $\square \square$

Software output consistent with input and with results reported in document reviewed.

Limits/criteria/guidelines applied to analysis results are appropriate and referenced.

Limits/criteria/guidelines checked against references.

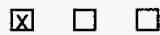

Safety margins consistent with good engineering practices.

区 $\square \square$

Conclusions consistent with analytical results and applicable limits.

Results and conclusions address all points required in the problem statement.

Format consistent with appropriate NRC Regulatory Guide or other standards.

$\begin{array}{lll}\square & \square & \square \\ \square & \square \quad \square\end{array}$

$\square \square$ 网

* Review calculations, comments, and/or notes are attached.

\ $\square \square$ Document approved.

M. G. Plys
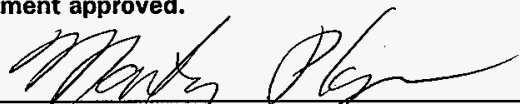

Reviewer (Printed Name and Signature)

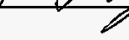

$07 / 23 / 98$

Date

*Any calculations, comments, or notes generated as part of this review should be signed, dated and attached to this checklist. Such material should be labeled and recorded in such a manner as to be intelligible to a technically qualified third party. 


\section{REVIEW CHECKLIST}

Document Reviewed:

"MCO Gas Composition for Low Reactive Surface Areas," HNF-3035, Rev. 0, M.J. Packer

Scope of Review:

Assumptions, Methodology, Conclusions

Yes No NA

区 $\square \square$

* Previous reviews complete and cover analysis, up to scope of this review, with no gaps.

网 Problem completely defined.

X $\square \square$

Accident scenarios developed in a clear and logical manner.

冈 $\square \square$

Necessary assumptions explicitly stated and supported.

冈 $\square \square$

Computer codes and data files documented.

$\mathrm{X}$

Data used in calculations explicitly stated in document.

$\mathrm{X}$

Data checked for consistency with original source information as applicable.

$\square \square \square$

Mathematical derivation checked including dimensional consistency of results.

$\mathrm{x}$

Models appropriate and used within range of validity or use outside range of established validity justified.

Hand calculations checked for errors. Spreadsheet results should be treated exactly the same as hand calculations.

Software input correct and consistent with document reviewed.

Software output consistent with input and with results reported in document reviewed.

Limits/criteria/guidelines applied to analysis results are appropriate and referenced.

Limits/criteria/guidelines checked against references.

Safety margins consistent with good engineering practices.

[ $\square \square$

网 $\square \square$

Conclusions consistent with analytical results and applicable limits.

回 $\square \square$

Results and conclusions address all points required in the problem statement.

Format consistent with appropriate NRC Regulatory Guide or other standards.

* Review calculations, comments, and/or notes are attached.

■ $\square \square$ Document approved.

R. A. Sexton

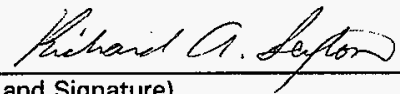

$07 / 22 / 98$

Reviewer (Printed Name and Signature)

Date

*Any calculations, comments, or notes generated as part of this review should be signed, dated and attached to this checklist. Such material should be labeled and recorded in such a manner as to be intelligible to a technically qualified third party. 


\subsection{INTRODUCTION AND PURPOSE}

Interim storage of multi-canister overpacks (MCOs) containing spent nuclear fuel (SNF) generates issues of increasing gas compositions within the MCO, notably oxygen, because of it's flammability properties. The safe storage of the SNF in MCOs can be demonstrated by using conservative conditions with modelled values. Current thought is to provide $0.5 \mathrm{~atm}$ overpressure of helium to the MCOs prior to the 40 year interim storage. However, using 'worst case' conservative conditions, such as low reactive surface areas on the uranium fuel, the modelled results (Duncan and Plys 1998) show the oxygen concentrations to be above the $4 \%$ limit. This document proves, using conservative conditions with a zero reactive surface area and backfilling the $\mathrm{MCO}$ with $1.5 \mathrm{~atm}$ overpressure ( $2.5 \mathrm{~atm}$ abs) of helium prior to interim storage, the MCOs can maintain oxygen concentration levels below 4 mole \%. Also, a hypothetical case exists with an MCO containing only $K$ East fuel with a range of little to none reactive surface areas and an initial backfill overpressure of $0.5 \mathrm{~atm}$ (1.5 atm abs). Again this document demonstrates, using modelled parameters, that this condition remains well below 4 mole $\%$ oxygen over the 40 year storage period.

\subsection{SCOPE}

The subject scope includes input values from modelling calculations in HNF-SD-SNF-TI-040, Rev. 2 (Duncan and Plys 1998) with a revised (increased) helium backfill pressure to demonstrate that oxygen mole (or volume) concentrations, after 40 years of MCO storage, do not exceed $4 \%$ over a range of low reactive surface areas. Conservative input variables are utilized, including a zero reactive surface area, along with a bounding uranium oxide hydrate value $\left(0.11 \mathrm{~kg} / \mathrm{m}^{2}\right)$ which is correlated with the amount of oxygen generated per reactive surface area via radiolytic decomposition. The major modelling input revision is the increase of the initial helium backfill pressure in the MCO from $0.5 \mathrm{~atm}$ to $1.5 \mathrm{~atm}$ overpressure (or total $2.5 \mathrm{~atm}$ absolute). In principal, any oxygen generation rate can be mitigated through an appropriate backfill pressure (Duncan and Plys 1998). Also considered is the case of only K East fuel with no reactive surface area at 1.5 atm absolute backfill pressure.

\subsection{KEY INPUTS AND ASSUMPTIONS}

Revisions to modelling calculations are included in Appendix A (re-formatted MATHCAD ${ }^{\mathrm{TM}}$ file from Duncan and Plys) and noted in bold face type. Modelled input parameters include the following as noted in Appendix A:

Decay Power: A range of MCO decay power values, from $10 \mathrm{~W}$ to $770 \mathrm{~W}$, is included in the calculations. An average MCO decay power is $396 \mathrm{~W}$.

Radiolysis: $\mathrm{Al}(\mathrm{OH})_{3}$ radiolysis is by gamma dose alone with $\mathrm{g}\left(\mathrm{H}_{2}\right)=1.2$ molecules $/ 100 \mathrm{eV}$ and $\mathrm{g}\left(\mathrm{O}_{2}\right)=0.225$ molecules $/ 100 \mathrm{eV}$. 
$\mathrm{UO}_{3} \cdot \mathrm{xH}_{2} \mathrm{O}$ radiolysis is by alpha, beta, and gamma sources with $\mathrm{g}\left(\mathrm{H}_{2}\right)=0.165,0.05$, and 1.2, respectively. $\mathrm{UO}_{3} \times \mathrm{xH}_{2} \mathrm{Og}\left(\mathrm{O}_{2}\right)$ values are $0.083,0.025$, and 0.11 for alpha, beta, and gamma, respectively.

Conservative values are included such as $8 \mathrm{~kg}$ aluminum hydroxide, $0.11 \mathrm{~kg}$ water $/ \mathrm{m}^{2}$ reactive surface area from uranium oxide hydrate, $200 \mathrm{~g}$ free water, and a range of low (and zero) reactive surface areas. The minimal to zero area is associated with minimal to no fuel scrap and undamaged fuel rods. Note that the 200 grams of free water remains as a constant over the range of reactive surface areas, including zero reactive area. This term is bounding since the amount of free water is associated with the cracks in the damaged fuel surface (reactive surface area). Helium backfill overpressure is increased from $0.5 \mathrm{~atm}$ to $1.5 \mathrm{~atm}$ ( $2.5 \mathrm{~atm} \mathrm{abs})$ prior to interim storage.

A separate conservative case is demonstrated assuming no $\mathrm{Al}(\mathrm{OH})_{3}$ with minimal to zero reactive surface areas at a helium backfill pressure of $1.5 \mathrm{~atm}$ absolute.

\subsection{CONCLUSION}

The plot in Appendix A, on page A-11, shows that use of an initial helium backfill pressure of $2.5 \mathrm{~atm}$ absolute in the MCO results in acceptable oxygen mole concentrations per low (and zero) reactive surface areas under conservative conditions. The 'worst case' condition of zero reactive surface area using this initial helium backfill pressure results in an oxygen concentration less than $4 \%$ over 40 years. A comparison plot, on page A-12, shows the results from an initial helium backfill overpressure of $0.5 \mathrm{~atm}(1.5 \mathrm{~atm}$ abs $)$ in an MCO using conservative input values. Page A-14 shows the results (oxygen below 3\%) from the hypothetical condition with an MCO filled with only $\mathrm{K}$ East fuel and minimal to zero reactive surface areas at an initial helium backfill pressure of $1.5 \mathrm{~atm}$ absolute.

\subsection{REFERENCE}

D. R. Duncan and M. G. Plys, 1998, "MCO Internal Gas Composition and Pressure During Interim Storage," HNF-SD-SNF-TI-040, Rev. 2, DE\&S Hanford, Richland, WA. 
MCO O2\% AND MAXIMUM PRESSURIZATION: RADIOLYSIS AND GETTERING OF O2

Goal is $\mathrm{O} 2$ concentration at end-of-life, or max concentration, so $\mathrm{H} 2$ gettering not considered.

RANGE OF Q AND A, DATABOOK G(O2) VALUES, 8 KG AL(OH)3,

BOUNDING $0.11 \mathrm{~kg} \mathrm{H} 2 \mathrm{O} / \mathrm{m}^{\wedge} 2$ FROM UO3.2H2O, 200 G RESIDUAL H2O (NO UO3.2H2O

DECOMP)

BY: Martin G. Plys, Fauske \& Associates, Inc. 16W070 W.83rd St.

Burr Ridge IL 60521 Phone 630-323-8750

FOR: Hanford Spent Nuclear Fuel Project - Duke Engineering \& Services Hanford

- Richland, WA

Contact: Darrell Duncan 509-372-1013.

DATE: June, 1998.

Calculation Technical Basis and Assumptions:

1. Decay power varies per HNF-SD-SNF-CN-006 Regulatory/Safety Design Basis:

$\begin{array}{llllcl}\text { source } & 1995 & 2040 & \text { lamda } & \text { tau } & \text { Where lamda in 1/year and tau in year } \\ \text { alpha } & 26.3 & 32 & -4.36 \mathrm{e}-3 & -159 & \text { Alpha increases as fraction with time } \\ \text { beta } & 65 & 22 & +2.41 \mathrm{e}-2 & 28.8 & \\ \text { gamma } & 42.4 & 14.3 & +2.42 \mathrm{e}-2 & 28.7 & \text { Note decay power in W/MT here } \\ \text { TOTAL } & 133.7 & 68.3 & 1.49 \mathrm{e}-2 & 46.4 & \end{array}$

2. Average MCO decay power is $396 \mathrm{~W}$, bounding power is $776 \mathrm{~W}$, and fuel mass is $6339 \mathrm{~kg}$. Alpha, beta, and gamma fractions from 1995 above are applied for all total MCO powers. So for example, alpha power in an average $\mathrm{MCO}$ is $=(26.3 / 133.7) * 396=77.9 \mathrm{~W}$.

3. MCO temperature is directly related to decay power as a function of time -- so $\mathrm{O} 2$ gettering is related to.

4. $\mathrm{Al}(\mathrm{OH}) 3$ radiolysis is by gamma dose alone with $\mathrm{g}(\mathrm{H} 2)=1.2$ molecules $/ 100 \mathrm{ev}$ [HNF-SNF-CN006 ]. $\mathrm{g}(\mathrm{O} 2)$ is either 0.225 [same ref] or 0.6 , conservative stoichiometric value. $\mathrm{A} / \mathrm{U}$ gamma absorption $=35 \%$.

5. Uranium Oxide Hydrates are represented by UO3.2H2O.

6. UO3.2H2O NOT ALLOWED TO DECOMPOSE THERMALLY - RESIDUAL H2O EVAPORATE.

7. UO3. $\mathrm{xH} 2 \mathrm{O}$ radiolysis is by alpha, beta, and gamma sources with $\mathrm{g}(\mathrm{H} 2)=0.165,0.05$ and 1.2 respectively, using the $11 \%$ water fraction when $x=2$ [HNF-SD-SNF-CN-006]. U hydrate/U gamma absorption $=86 \%$.

8. UO3. $\mathrm{xH} 2 \mathrm{O} \mathrm{g}(\mathrm{O} 2)$ values are: $0.083,0.025$, and 0.11 for alpha, beta, gamma respectively from [same ref], but a gamma value of 0.6 is a conservative stoichiometric value.

9. No hydrogen gettering - only occurs when Oxygen is depleted. This is OK because it allows the maximum presssure to be calculated, before substantial $\mathrm{H} 2$ gettering, and the max. $\mathrm{O} 2$ concentration.

10. Oxygen gettering by Ritchie's moist air correlation, since $\mathrm{H} 2 \mathrm{O}$ present, with a minimum limit of Trimble's dry air correlation. 
11. Bounding values are: $1.5 \mathrm{~atm}$ backfill overpressure, $2.8 \mathrm{~kg}$ water from $\mathrm{Al}(\mathrm{OH}) 3$ with no removal, $0.77 \mathrm{~kg}$ water from UO3.2H2O $7 \mathrm{~m}^{\wedge} 2$. Inputs may differ from bounds. Best-estimate is $0.055 \mathrm{~kg} \mathrm{H} 2 \mathrm{O} / \mathrm{m}^{\wedge} 2$ from $\mathrm{UO} 3.2 \mathrm{H} 2 \mathrm{O}$; bound is $0.11 \mathrm{~kg} / \mathrm{m}^{\wedge} 2$. Bounding area is $8 \mathrm{~m}^{\wedge} 2$.

** Note on units: time in years, mass in grams, area in $m^{\wedge} 2$, with appropriate conversions used.

\subsection{INPUT AND DERIVED VALUES}

Avogadro's number:

Conversion factor $\mathrm{J} / 100 \mathrm{ev}$ :

MCO volume, $\mathrm{m}^{\wedge} 3$,

and backfill temperature, $\mathrm{K}$ :

Fuel Mass (grams):

UO3 Hydrate mass per unit area gram $/ \mathrm{m}^{\wedge} 2$ :

Max $\mathrm{g}$ H2O from $\mathrm{UO} 3 . x \mathrm{H} 2 \mathrm{O} \& \max \mathrm{g} \mathrm{Al}(\mathrm{OH}) 3$ :

Free $\mathrm{H} 2 \mathrm{O}$, g, bounding value:

Amount of water from $\mathrm{UO} 4.2 \mathrm{H} 2 \mathrm{O}$ if no $\mathrm{AL}(\mathrm{OH}), \mathrm{g}$ :

Molecular weights of $\mathrm{Al}(\mathrm{OH}) 3$ and $\mathrm{UO} 3$ hydrate:

$\mathrm{g}(\mathrm{H} 2) \& \mathrm{~g}(\mathrm{O} 2)$ value for $\mathrm{Al}(\mathrm{OH}) 3$, molec/100 eV:

$\mathrm{g}(\mathrm{H} 2)$ values for $\mathrm{UO} 3$ hydrates, molec/100 eV

$\mathrm{g}(\mathrm{O} 2)$ values for $\mathrm{UO} 3$ hydrates, molec $/ 100 \mathrm{eV}$

$\mathrm{g}(\mathrm{H} 2)$ values for free $\mathrm{H} 2 \mathrm{O}$, molec $/ 100 \mathrm{eV}$

$$
\begin{aligned}
& \mathrm{Na}:=6.022 \cdot 10^{23} \\
& \mathrm{JeV}:=1.6 \cdot 10^{-17} \\
& \mathrm{~V}_{\mathrm{mco}}=0.5 \\
& \mathrm{~T}_{\mathrm{bf}}=298 \\
& \mathrm{M}_{\mathrm{f}}:=6.33910^{6} \\
& \mathrm{~m}=110 \quad \text { BOUNDING } 0.11 \mathrm{~kg} / \mathrm{m}^{\wedge} 2 ! \\
& \mathrm{UH}_{\max }=770 \\
& \mathrm{AL}_{\max }=8000
\end{aligned}
$$

$\mathrm{m}_{\mathrm{fw}}=200$

$$
\mathrm{m}_{\mathrm{u} 04}=54
$$

$$
\begin{aligned}
& M_{\text {ah }}=78 \\
& M_{\text {uh }}=322 \\
& M_{w}=18
\end{aligned}
$$

$$
\begin{aligned}
& \mathrm{g}_{\text {ahy }}=1.2 \\
& \mathrm{~g}_{\text {ougy }}=0.225 \\
& \mathrm{~g}_{\text {hux }}=0.165 \\
& \mathrm{~g}_{\text {hup }}=0.05 \\
& \mathrm{~g}_{\text {huy }}=1.2 \\
& \mathrm{~g}_{\text {oud }}=0.083 \\
& \mathrm{~g}_{\text {ouß }}=0.025 \\
& \mathrm{~g}_{\text {oury }}=0.11
\end{aligned}
$$

$$
\begin{aligned}
& \mathrm{g}_{\mathrm{hw \alpha}}=1.6 \\
& \mathrm{~g}_{\mathrm{hw \beta}}=0.53 \\
& \mathrm{~g}_{\mathrm{hw \gamma}}=0.5
\end{aligned}
$$


HNF-3035, Rev. 0

Appendix A

$\mathrm{g}(\mathrm{O} 2)$ values for free $\mathrm{H} 2 \mathrm{O}$, molec $/ 100 \mathrm{eV}$

$$
\begin{aligned}
& \mathrm{g}_{\mathrm{ow} \alpha}=0.8 \\
& \mathrm{~g}_{\mathrm{ow} \beta}=0.265 \\
& \mathrm{~g}_{\mathrm{owy}}=0.25
\end{aligned}
$$

Relative gamma absorption for $\mathrm{Al}, \mathrm{U}, \mathrm{H} 2 \mathrm{O}$ :

$$
\begin{aligned}
& \mathrm{rQ}_{\mathrm{ay}}=0.35 \\
& \mathrm{rQu}_{\mathrm{uy}}=0.86 \\
& \mathrm{rQ}_{w y}=0.38
\end{aligned}
$$

Power fraction for $\mathrm{Al}(\mathrm{OH}) 3$. Multiplies initial power; decays with time per below.

$$
\begin{aligned}
& \mathrm{fQ}_{\mathrm{xy}}=r \mathrm{Q}_{\mathrm{dy}} \cdot \underline{42.4} \\
& 133.7 \\
& \mathrm{fQ}_{\mathrm{xy}}=0.111 \\
& \mathrm{fQ}_{\mathrm{u} \alpha}=\underline{26.3} \\
& 133.7 \\
& \mathrm{fQ}_{\mathrm{u \beta}}=\underline{65} \\
& 133.7 \\
& \mathrm{fQ}_{\mathrm{ur}}=\mathrm{rQ}_{\mathrm{ur}} \underline{42.4}
\end{aligned}
$$

Power fractions for $\mathrm{U}$ hydrates, Each independently decayed with time.

$$
\begin{aligned}
& \mathrm{fQ}_{\mathrm{u \alpha}}=0.1967 \\
& \mathrm{fQ}_{\mathrm{u} \beta}=0.4862 \\
& \mathrm{fQ}_{\mathrm{u \gamma}}=0.2727
\end{aligned}
$$

Power fractions for for free $\mathrm{H} 2 \mathrm{O}$, each independently decayed with time.

$$
\begin{aligned}
& \mathrm{fQ}_{w \alpha}:=\mathrm{fQ}_{\mathrm{u} \alpha} \\
& \mathrm{fQ}_{\mathrm{w \beta}}:=\mathrm{fQ}_{\mathrm{u} \beta} \\
& \mathrm{fQ}_{\mathrm{wy}}=\mathrm{rQ}_{w y} \frac{42.4}{133.7} \\
& \mathrm{fQ}_{\mathrm{wy}}=0.1205
\end{aligned}
$$

Decay rates for alpha,beta,gamma, and total power based on 1995 to 2040 changes. Note alpha power increases slightly, so rate is negative.

$$
\begin{aligned}
& \lambda_{\alpha}:=\frac{-1}{45} \cdot \ln \left(\frac{32}{26.3}\right) \\
& \lambda_{\alpha}=-4.359310^{-3} \\
& \lambda_{\beta}:=\frac{-1}{45} \cdot \ln \left(\frac{22}{65}\right) \\
& \lambda_{\beta}=0.0241
\end{aligned}
$$




$$
\begin{aligned}
& \lambda_{q}:=\frac{-1}{45} \cdot \ln \left(\frac{68.3}{133.7}\right) \\
& \lambda_{q}=0.0149 \\
& \lambda_{\gamma}:=\frac{-1}{45} \cdot \ln \left(\frac{14.3}{42.4}\right) \\
& \lambda_{\gamma}=0.0242
\end{aligned}
$$

\subsection{FUNCTIONS FOR REMAINING MASS DUE TO RADIOLYSIS}

2.1.1 $\mathrm{Al}(\mathrm{OH}) 3$ radiolysis fractions and example for $396 \mathrm{~W}$ in $6339 \mathrm{~kg}$ (W/g unit used):

$$
\begin{aligned}
& \lambda_{\text {ao }}:=\frac{2}{3} \cdot \mathrm{M}_{\mathrm{f}}^{-1} \cdot \mathrm{M}_{\mathrm{ah}} \cdot(\mathrm{Jev} \cdot \mathrm{Na})^{-1} \cdot(360024 \cdot 365) \\
& \lambda_{\mathrm{ao}}=2.684910^{-5} \\
& \Lambda_{\mathrm{a}}(\mathrm{Q}, \mathrm{t}):=\frac{\lambda_{\mathrm{ao}} \cdot \mathrm{g}_{\mathrm{ha \gamma}} \cdot \mathrm{fQ}_{\mathrm{a \gamma}} \cdot \mathrm{Q}}{\lambda_{\gamma}} \cdot\left(\mathrm{e}^{-\lambda_{\gamma} \mathrm{t}}-1\right) \\
& \mathrm{F}_{\mathrm{a}}(\mathrm{Q}, \mathrm{t}):=\mathrm{e}^{\Lambda_{\mathrm{a}}(\mathrm{Q}, \mathrm{t})}
\end{aligned}
$$

Fraction (mass or moles) $\mathrm{Al}(\mathrm{OH}) 3$ left as function of $\mathrm{MCO}$ power, time

$$
\mathrm{F}_{\mathrm{a}}(396,40)=0.9643
$$

Fraction left in average MCO after 40 years: $3.6 \%$ decomposition.

$$
\mathrm{dF}_{h a}(\mathrm{Q}, \mathrm{t}):=-\lambda_{\text {ao }} \cdot \mathrm{g}_{\text {hay }} \cdot f \mathrm{Q}_{\mathrm{ay}} \cdot \mathrm{Q} \cdot \mathrm{e}^{-\lambda} \gamma^{\mathrm{t}}
$$

Derivative of fraction: Used for $\mathrm{H} 2$ radiolysis rate

$$
\mathrm{dF}_{h a}(396,20)=-8.736 \times 10^{-4}
$$

About $-0,09 \%$ per year implies $-3.6 \%$ over 40 years

$$
\mathrm{dF}_{\mathrm{oa}}(\mathrm{Q}, \mathrm{t}):=-\lambda_{\mathrm{ao}} \cdot \mathrm{g}_{\mathrm{oay}} \cdot \mathrm{PQ}_{\mathrm{ay}} \cdot \mathrm{Q} \cdot \mathrm{e}^{-\lambda \gamma^{t}}
$$

Derivative of fraction: Used for $\mathrm{O} 2$ radiolysis rate

2.1.2 UO3.2H2O radiolysis fractions and example for $396 \mathrm{~W}$ :

$$
\begin{aligned}
& \lambda_{\text {uo }}:=M_{f}^{-1} \cdot M_{\text {uh }} \cdot(\text { Jev } \cdot N a)^{-1} \cdot(3600 \cdot 24 \cdot 365) \\
& \lambda_{\text {uo }}=1.662610^{-4}
\end{aligned}
$$

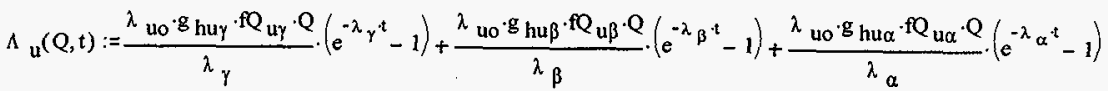

$$
\begin{aligned}
& \mathrm{F}_{u}(\mathrm{Q}, \mathrm{t}):=\mathrm{e}^{\wedge_{u}(\mathrm{Q}, \mathrm{t})}
\end{aligned}
$$


Fraction (mass or moles) $\mathrm{U}$ hydrate left as function of MCO power, time

$$
F_{u}(396,40)=0.503
$$

Fraction left in average $\mathrm{MCO}$ after 40 years: $50 \%$ decomposition.

Derivative of fraction for $\mathrm{H} 2$ radiolysis rate:

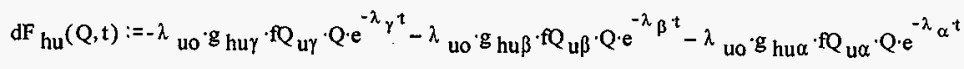

$$
\begin{aligned}
& \mathrm{dF}_{h u}(396,20)=-0.0166
\end{aligned}
$$

About $-1.6 \%$ per year implies $>50 \%$ over 40 years (rate varies strongly) 02 production from radiolysis of UO3.2H2O:

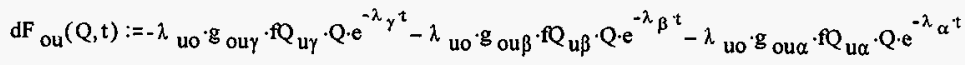

\subsubsection{Free water decomposition}

$\lambda_{\text {wo }}:=\mathrm{M}_{\mathrm{f}}^{-1} \cdot \mathrm{M}_{\mathrm{w}} \cdot(\mathrm{Jev} \cdot \mathrm{Na})^{-1} \cdot(360024 \cdot 365)$

$\lambda_{\text {wo }}=9.293910^{-6}$

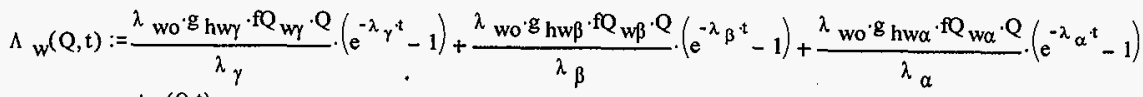

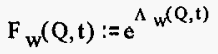

Fraction (mass or moles) U hydrate left as function of MCO power, time

$F_{W}(396,40)=0.9225$

Fraction left in average MCO after 40 years: $8 \%$ decomposition.

Derivative of fraction for $\mathrm{H} 2$ radiolysis rate:

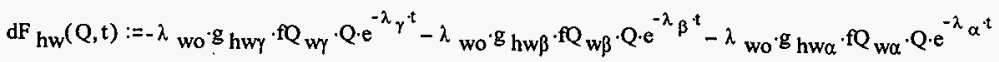

$\mathrm{dF}_{\mathrm{hw}}(396,20)=-1.986610^{-3}$

About $-0.2 \%$ per year implies $8 \%$ over 40 years $\mathrm{O} 2$ production from radiolysis of free $\mathrm{H} 2 \mathrm{O}$

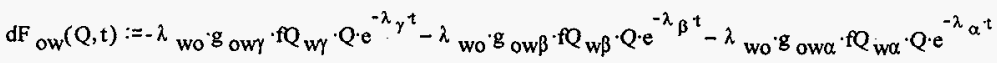


HNF-3035, Rev. 0

Appendix A

\subsection{FUNCTIONS FOR U GETTERING RATE AND WATER VAPOR PRESSURE}

Water vapor pressure:

$\mathrm{fP}_{\text {sat }}(\mathrm{T}):=\mathrm{e}^{\left(25.339-\frac{5154.7}{\mathrm{~T}}\right)}$

$\mathrm{fP}_{\text {sat }}(323)=1.185 \times 10^{4} \quad 50 \mathrm{C} \mathrm{OK}$

Ritchie correlation for U-H2O-O2, below $100 \%$ RH, below $100 \mathrm{C}$, agrees best with data BUT goes below dry air correlation at about $37 \mathrm{C}$ - so switch to McGillivray dry air. Ritchie units of $\mathrm{mg} / \mathrm{cm}^{\wedge} 2 / \mathrm{hr}$, converted to grams $\mathrm{O} 2$ per $\mathrm{m}^{\wedge} 2$ per year.; McGillivray units of $\mathrm{kg} / \mathrm{m}^{\wedge} 2 / \mathrm{s}$ similarly converted.

$\mathrm{K}_{\mathrm{rit}}(\mathrm{T}):=10^{13.8808-5769.6 \cdot \mathrm{T}^{-1}} \cdot 10.876 \quad$ Ritchie

$K_{\text {trim }}(T):=10^{7.19-3732 \cdot T^{-1}} \cdot 10.876 \quad$ Trimble

$T_{\text {cor }}:=(5769.6-3732) \cdot(13.8808-7.19)^{-1}$

$T_{\text {cor }}=304.5376$

Switch correlations here

$\mathrm{fK}_{\mathrm{o}}(\mathrm{T}):=\mathrm{if}\left(\mathrm{T} \geq \mathrm{T}\right.$ cor $\left., \mathrm{K}_{\text {rit }}(\mathrm{T}), \mathrm{K}_{\text {trim }}(\mathrm{T})\right)$

$\mathrm{fK}_{\mathrm{o}}(323) \cdot 0.1 \cdot(876)^{-1}=1.042910^{-4} \quad$ OK @ $50 \mathrm{C}$ in $\mathrm{mg} / \mathrm{cm}^{\wedge} 2 / \mathrm{hr} \quad$ Shows no discontinuity

$\mathrm{fK}_{\mathrm{o}}(323) \cdot 10^{-3} \cdot\left(3.15 \cdot 10^{7}\right)^{-1}=2.902410^{-10} \quad \mathrm{OK} @ 50 \mathrm{C}$ in $\mathrm{kg} / \mathrm{m}^{\wedge} 2 / \mathrm{s}$

$\mathrm{fK}_{\mathrm{o}}\left(\mathrm{T}_{\mathrm{cor}}+0.01\right) \cdot 10^{-3} \cdot\left(3.15 \cdot 10^{7}\right)^{-1}=2.401410^{-11}$

$\mathrm{fK}_{\mathrm{o}}\left(\mathrm{T}_{\mathrm{cor}}-0.01\right) \cdot 10^{-3} \cdot\left(3.15 \cdot 10^{7}\right)^{-1}=2.395810^{-11}$

\subsection{RELATIONSHIP BETWEEN MCO POWER AND GAS TEMPERATURE}

For $396 \mathrm{~W} \mathrm{MCO,} \mathrm{Conservative} \mathrm{low} \mathrm{temperature} \mathrm{drops} \mathrm{are:}$

$13 \mathrm{C}$ delta- $\mathrm{T}$ vault gas to Tube wall; $15 \mathrm{C}$ across gap; $3 \mathrm{C}$ to scrap average;

so hmax for delta-Tmin: $396 /(13+15+3)=396 / 31=12.8$. Conservative value is $13 \mathrm{~W} / \mathrm{K}$.

Min. Getter temperature, given MCO power, vault entrance

$F \operatorname{Tmin}\left(Q_{0}, t\right)=\underline{Q}_{0} \cdot \frac{\exp \left(-\lambda_{9} \cdot t\right)}{13}+12$ 
2.4 HYDRATE PARTIAL DECOMPOSITION DETERMINES INITIAL PRESSURE, INVENTORY

*** Residual free water assumed to prevent UO3.2H2O decomposition ***

NOTE: Area used by these functions is fuel reactive area, NOT any additional getter area.

$\mathrm{N}_{\mathrm{wa}}\left(\mathrm{m}_{\mathrm{ah}}\right):=1.5 \cdot \mathrm{m}_{\mathrm{ah}} \cdot \mathrm{M}_{\mathrm{ah}}{ }^{-1}$

Aluminum hydroxide water moles

Function for moles of UO3.2HO plus $\mathrm{UO} 4.2 \mathrm{H} 2 \mathrm{O}$ givem $\mathrm{m}=\mathrm{kg} / \mathrm{m}^{\wedge} 2$ from $\mathrm{UO} 3.2 \mathrm{H} 2 \mathrm{O}, \mathrm{A}=$ fuel reactive area, and adding UO4 contribution in proportion the fraction not covered with $\mathrm{Al}(\mathrm{OH}) 3$, given its mass ma.

$n_{w u}\left(m, A, m_{a}\right):=\left[i\left\{(m \cdot A)>U_{\max } U_{\max } m \cdot A\right]+m_{u o 4} \cdot\left(1-\frac{m_{a}}{A L_{\max }}\right)\right] \frac{1}{M_{w}}$

$\mathrm{N}_{w u}\left(\mathrm{Q}, \mathrm{A}, \mathrm{m}_{\mathrm{a}}\right):=\mathrm{n}_{\text {wu }}\left(\mathrm{m}, \mathrm{A}, \mathrm{m}_{\mathrm{a}}\right)$

Hydrate moles - NO adjustment for thermal decomposition

$\mathrm{N}_{\mathrm{st}}(\mathrm{Q}):=\mid \begin{aligned} & \mathrm{T}_{\mathrm{o}} \leftarrow \mathrm{fTmin}(\mathrm{Q}, 0)+273 \\ & \mathrm{fP}_{\text {sat }}\left(\mathrm{T}_{\mathrm{o}}\right) \cdot \mathrm{V}_{\mathrm{mco}} \cdot\left(8.314 \mathrm{~T}_{\mathrm{o}}\right)^{-1}\end{aligned}$

Initial steam from free water, Assumed sufficient!

$\mathrm{N}_{\mathrm{fw}}:=\mathrm{m}_{\mathrm{fw}} \cdot \mathrm{M}_{\mathrm{w}}{ }^{-1}$

Initial free water moles, No adjustment for evaporation

\subsection{FUNCTIONS FOR IRON GETTER}

Parabolic kinetic rate law, Units are $\mathrm{m}^{\wedge} 2 / \mathrm{yr}$. Answer at $50 \mathrm{C}$ is $1.9^{*} 10^{\wedge}-19 \mathrm{~cm}^{\wedge} 2 / \mathrm{s}$, or $6 \mathrm{e}-16 \mathrm{~m}^{\wedge} 2 / \mathrm{yr}$. If Mathcad says zero, then change 'zero tolerance' in menu

$\mathrm{fK}_{\mathrm{Fe}}(\mathrm{T}):=3.15 \cdot 10^{7} \cdot 10^{-4} \cdot 5.566310^{-11} \cdot \mathrm{e}^{-6295.6 \cdot \mathrm{T}^{-1}}$

$\mathrm{fK}_{\mathrm{Fe}}(323)=6.012210^{-16}$

Getter area, $\mathrm{m}^{\wedge} 2$, given current moles and net molar production rate

$\mathrm{fA}_{\text {get }}\left(\mathrm{n}_{\mathrm{o} 2}, \mathrm{dn} \mathrm{o}_{2}, \mathrm{~T}\right):=10^{-6} \cdot \frac{55.85}{7.8} \cdot \frac{3}{2} \cdot \sqrt{2 \cdot \mathrm{n}_{\mathrm{o} 2} \cdot \mathrm{dn}_{\mathrm{o} 2} \cdot \mathrm{fK}_{\mathrm{Fe}}(\mathrm{T})^{-1}}$

3.0 FUNCTIONS FOR GAS EVOLUTION WITH TIME, MCO POWER, GETTER AREA, AI(OH)3:

$3.1 \mathrm{H} 2+\mathrm{H} 2 \mathrm{O}$ : With no hydrogen gettering, $\mathrm{H} 2+\mathrm{H} 2 \mathrm{O}$ is a function of time until $\mathrm{O} 2$ runs out:

$$
\mathrm{fN}_{h 2}\left(Q, A, m_{a}, t\right):=N_{s t}(Q)+N_{w u}\left(Q, A, m_{a}\right) \cdot\left(1-F_{u}(Q, t)\right)+N_{w a}\left(m_{a}\right) \cdot\left(1-F_{a}(Q, t)\right)+N_{f w} \cdot\left(1-F_{w}(Q, t)\right)
$$


3:2 O2: Oxygen gettering depends upon time-dependent $\mathrm{MCO}$ temperature, and must be integrated. Need a trick because Mathcad demands a function $F$ whose arguments are $t, Y$ where $t$ is time and $Y$ is a vector of state variables. Output of $F$ is a vector $\mathrm{dY} / \mathrm{dt}$. Trick: $\mathrm{dY} / \mathrm{dt}=0$ for constant terms needed.

$$
\begin{aligned}
& \operatorname{dO} 2 \operatorname{dt}(t, Y):=\mid \begin{array}{l}
\mathrm{Y}-\mathrm{Y}_{0} \\
\mathrm{~A} \leftarrow \mathrm{Y}_{1}
\end{array} \\
& m_{a} \leftarrow Y_{2} \\
& \mathrm{R} \leftarrow \mathrm{Y}_{3} \\
& \mathrm{Y}_{4} \leftarrow \text { if }\left[\left(\mathrm{Y}_{4}<0\right), 0, \mathrm{Y}_{4}\right] \\
& \mathrm{N}_{\mathrm{O} 2} \leftarrow \mathrm{Y}_{4} \\
& N_{u} \leftarrow N_{w u}\left(Q, A, m_{a}\right) \cdot F_{u}(Q, t) \\
& N_{a} \leftarrow N_{w a}\left(m_{a}\right) \cdot F_{a}(Q, t) \\
& \mathrm{N}_{w}{ }^{-N_{f w}} \mathrm{~F}_{w}(\mathrm{Q}, \mathrm{t}) \\
& \text { sorca }--d F_{o a}(Q, t) \cdot N_{a} \\
& \text { sorcu } \leftarrow-\mathrm{dF}_{\text {ou }}(\mathrm{Q}, \mathrm{t}) \cdot \mathrm{N}_{\mathrm{u}} \\
& \text { sorcw }-d F \text { ow }(Q, t) \cdot \mathrm{N}_{\mathrm{w}} \\
& T_{\text {get }} \leftarrow \text { fTmin }(Q, t)+273 \\
& \text { sink } \leftarrow(\mathrm{R} \cdot \mathrm{A}) \cdot \mathrm{fK} \mathrm{o}_{\mathrm{o}}\left(\mathrm{T}_{\text {get }}\right) \cdot 32^{-1} \\
& \text { net }- \text { sorca + sorcu + sorcw - sink } \\
& \text { dO2dt } \leftarrow \mid \begin{array}{l}
\text { net if }\left(\mathrm{N}_{\mathrm{o} 2}>0\right)+(\text { net }>0) \\
0 \text { otherwise }
\end{array}
\end{aligned}
$$

First, assign scrutable names to elements of $Y$ :

$\mathrm{Q}=$ Power, $\mathrm{A}=$ Fuel Area, $\mathrm{ma}=\mathrm{Al}(\mathrm{OH}) 3$ mass,

$\mathrm{R}=$ Rate law multiplier, $\mathrm{No} 2=\mathrm{O} 2$ moles

Make $\mathrm{O} 2$ moles $>0$ for numerical purposes. Current $\mathrm{UO} 3$ hydrate, $\mathrm{Al}(\mathrm{OH}) 3$, and free water moles Current source rates from radiolysis Current gettering rate by $U$ surfaces Net rate includes sink when there is $\mathrm{O} 2$ present, or at least when the rate is positive (net rate is zero when no $\mathrm{O} 2$ and sink exceeds source). Definition of rate of change: Here the trick is used, $\mathrm{dY} / \mathrm{dt}=0$ for constants

$F(t, Y):=\left(\begin{array}{llllll}0 & 0 & 0 & 0 & d O 2 d t(t, Y)\end{array}\right)^{T}$

Integrating function to just get $\mathrm{O} 2$ moles at $\mathrm{t}$ years:

Test function for average $\mathrm{MCO}$, bound $\mathrm{Al}(\mathrm{OH}) 3$ : 


$$
\mathrm{fN}_{\mathrm{o} 2}\left(\mathrm{Q}, \mathrm{A}, \mathrm{m}_{\mathrm{a}}, \mathrm{R}, \mathrm{t}\right):=\mid \begin{aligned}
& \mathrm{Y} \leftarrow\left[\mathrm{Q} \text { A } \mathrm{m}_{\mathrm{a}} \mathrm{R} \quad 30 \cdot 10^{-5}\right]^{\mathrm{T}} \\
& \text { last } \leftarrow \mathrm{t} \\
& \mathrm{Z}_{\leftarrow} \mathrm{Rkadapt}(\mathrm{Y}, \mathrm{O}, \mathrm{t}, \text { last }, \mathrm{F}) \\
& \left(\mathrm{Z}^{\langle S\rangle}\right)_{\text {last }}
\end{aligned}
$$

$\mathrm{N}_{\mathrm{o} 2}(400,1,8000,1,40)=0.3075$

$\mathrm{fN}_{\mathrm{o} 2}(400,2,8000,1,40)=7.4381 \cdot 10^{-3}$

Conclusion: Average MCO will NOT have oxygen buildup!

4.0 RESULTS: 8 AL(OH)3, $0.11 \mathrm{~kg} / \mathrm{m}^{\wedge} 2$, RATE MULT=1, $2.5 \mathrm{~atm}$ total from He backfil

$$
\mathrm{m}_{\mathrm{a}}=8000 \quad \mathrm{Al}(\mathrm{OH}) 3 \text { bounding mass, grams }
$$

$\mathrm{R}=1.0 \quad$ Rate law multiplier

Parametric variation for various power and getter areas:

Power 10 to $770 \mathrm{~W}$, general increments of 50 in the range,

Area up to $5 \mathrm{~m}^{\wedge} 2$ with 0.25 increments below 1.5

$\mathrm{Q}=16 \quad \mathrm{Q}_{\mathrm{q}}:=50 \cdot \mathrm{q} \quad \mathrm{Q}_{0}=10 \cdot \mathrm{Q}_{16}=770$

The reactive surface area range is at $0,0.005,0.01,0.025,0.05$, and $0.1 \mathrm{~m}^{\wedge} 2$

a $:=0 ., 5$

$$
A:=\left(\begin{array}{llllll}
0 & 0.005 & 0.01 & 0.025 & 0.05 & 0.1
\end{array}\right)^{\mathrm{T}}
$$

Hydrogen:

$$
\mathrm{nh} 2_{\mathrm{q}, \mathrm{a}}:=\mathrm{fN}_{\mathrm{h} 2}\left(\mathrm{Q}_{\mathrm{q}}, \mathrm{A}_{\mathrm{a}}, \mathrm{m}_{\mathrm{a}}, 40\right)
$$

Oxygen:

$$
\mathrm{no2}_{\mathrm{q}, \mathrm{a}}:=\mathrm{fN}_{\mathrm{o} 2}\left(\mathrm{Q}_{\mathrm{q}}, \mathrm{A}_{\mathrm{a}}, \mathrm{m}_{\mathrm{a}}, \mathrm{R}, 40\right)
$$

Helium backfill:

$$
\mathrm{P}_{\mathrm{bf}}=2.5
$$

$$
\begin{aligned}
& \mathrm{n}_{\text {he }}:=\mathrm{P}_{\text {bf }} 10^{5} \cdot \mathrm{V}_{\text {mco }}\left(8.314 \mathrm{~T}_{\mathrm{bf}}\right)^{-1} \\
& \mathrm{n}_{\text {he }}=50.4526
\end{aligned}
$$

Functions for concentration and pressure:

$$
\begin{aligned}
& \text { ntot }_{q, a}:=n_{h e}+n_{q, a}+2_{q, a} \\
& 02 \%_{a, q}:=\text { no2 }_{q, a} \cdot\left(\text { ntot }_{q, a}\right)^{-1}
\end{aligned}
$$


HNF-3035, Rev. 0

Appendix A

O2 Mass in grams

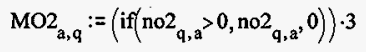

$$
\begin{aligned}
& 02 \%_{a, q}:=i f\left(O 2 \%_{a, q}>0,100-02 \%_{a, q}, 0\right)
\end{aligned}
$$

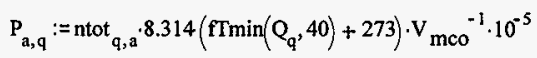

$\mathrm{O} 2$ Generation rate at time t, grams/year GIVEN calculation of no2 already (above) as an input:

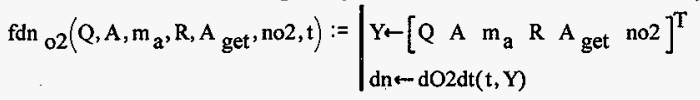

Required getter Area, $\mathrm{m}^{\wedge} 2$ :

$$
A F E_{a, q}:=f A_{g e t}\left(\frac{M O 2_{a, q}}{32}, f d n o 2\left(Q_{q}, A_{a}, m_{a}, R, 0, \frac{M O 2_{a, q}}{32}, 40\right), f \min \left(Q_{q}, 40\right)+273\right)
$$


Oxygen Percentage at 40 Years as a Function of MCO Power for Various Reactive Surface Areas, Case of: $8 \mathrm{~kg} \mathrm{Al}(\mathrm{OH}) 3,0.11 \mathrm{~kg} / \mathrm{m}^{\wedge} 2$ water from UO3.2H2O, $200 \mathrm{~g}$ Free H2O, BF Helium at 2.5 atm

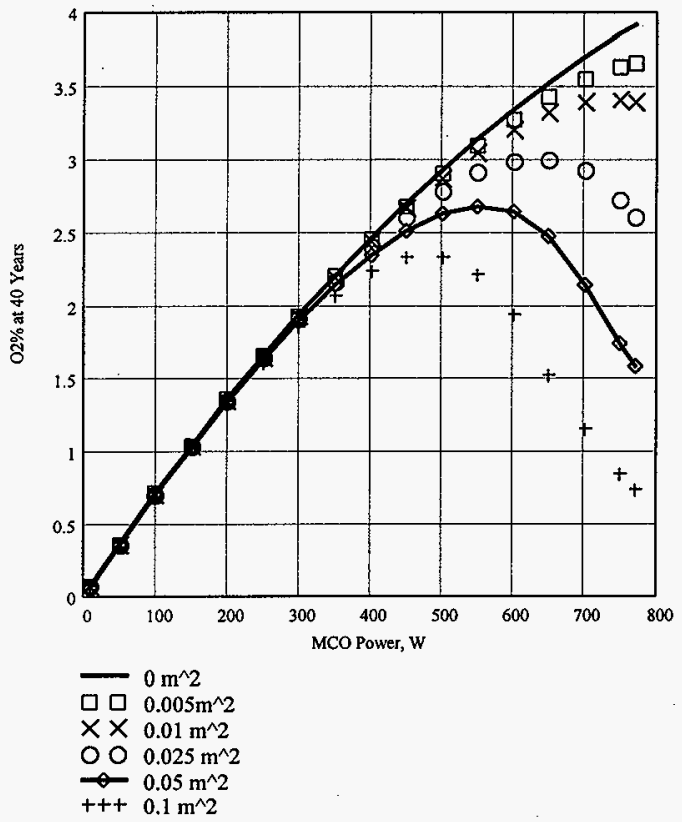

RESULTS : $\quad 8 \mathrm{~kg} \mathrm{AL}(\mathrm{OH}) 3,0.11 \mathrm{~kg} / \mathrm{m}^{\wedge} 2, \mathrm{RATE}$ MULT $=1.0,2.5$ atm He BACKFILL Oxygen concentration as function of area, $\mathrm{m}^{\wedge} 2$ (row=constant $\mathrm{A}$ ) and power, $W$ (column=constant $Q$ )

\begin{tabular}{|c|c|c|c|c|c|c|c|c|c|c|c|c|c|c|c|c|c|c|}
\hline & $Q$ & 10 & 50 & 100 & 150 & 200 & 250 & 300 & 350 & 400 & 450 & 500 & 550 & 600 & 650 & 700 & 750 & 770 \\
\hline A & $\because$ & 3 & & o & 4 & 3 & H. & $M$ & 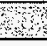 & $\mathrm{SH}$ & 35 & 35 & 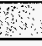 & 3 & 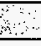 & & & \\
\hline 0 & & 0.1 & 0.4 & 0.7 & 1 & 1.4 & 1.7 & 1.9 & 2.2 & 2.5 & 2.7 & 2.9 & 3.1 & 3.3 & 3.5 & 3.7 & 3.9 & 3.9 \\
\hline .005 & 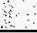 & 0.1 & 0.4 & 0.7 & 1 & 1.4 & 1.6 & 1.9 & 2.2 & 2.4 & 2.7 & 2.9 & 3.1 & 3.3 & 3.4 & 3.5 & 3.6 & 3.7 \\
\hline .01 & $y$ & 0.1 & 0.4 & 0.7 & 1 & 1.4 & $1: 6$ & 1.9 & 2.2 & 2.4 & 2.7 & 2.9 & 3.1 & 3.2 & 3.3 & 3.4 & 3.4 & 3.4 \\
\hline .025 & $\pi$ & 0.1 & 0.4 & 0.7 & 1 & 1.3 & 1.6 & 1.9 & 2.2 & 2.4 & 2.6 & 2.8 & 2.9 & 3 & 3 & 2.9 & 2.7 & 2.6 \\
\hline .05 & 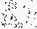 & 0.1 & 0.4 & 0.7 & 1 & 1.3 & 1.6 & 1.9 & 2.1 & 2.3 & 2.5 & 2.6 & 2.7 & 2.6 & 2.5 & 2.1 & 1.7 & 1.6 \\
\hline 0.1 & 4 & 0 & 0.3 & 0.7 & 1 & 1.3 & 1.6 & 1.9 & 2.1 & 2.2 & $2.3^{\circ}$ & 2.3 & 2.2 & 1.9 & 1.5 & 1.2 & 0.8 & 0.7 \\
\hline
\end{tabular}


HNF-3035, Rev. 0

Appendix A

MCO Pressure (atm abs) Over 40 Years, $A$ in units of $\mathrm{m}^{2}, Q$ in units of Watts

\begin{tabular}{|c|c|c|c|c|c|c|c|c|c|c|c|c|c|c|c|c|c|c|}
\hline & $Q$ & 10 & 50 & 100 & 150 & 200 & 250 & 300 & 350 & 400 & 450 & 500 & 550 & 600 & 650 & 700 & 750 & 770 \\
\hline A & 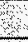 & 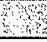 & 18 & Hat & 3 & mas & 4 & Hid & 3 & 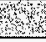 & Xy & 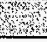 & $2 \%$ & $5^{2} 3$ & Wh & 3 & 4 & s \\
\hline 0 & & \begin{tabular}{|l}
2.4 \\
\end{tabular} & 2.5 & 2.5 & 2.6 & 2.7 & 2.8 & 2.9 & 3 & 3.1 & 3.2 & 3.3 & 3.4 & 3.5 & 3.5 & 3.6 & 3.7 & 3.7 \\
\hline .005 & & 2.4 & 2.5 & 2.5 & 2.6 & 2.7 & 2.8 & 2.9 & 3 & 3.1 & 3.2 & 3.3 & 3.4 & 3.5 & 3.5 & 3.6 & 3.7 & 3.7 \\
\hline .01 & $\cdots$ & 2.4 & 2.5 & 2.5 & 2.6 & 2.7 & 2.8 & 2.9 & 3 & 3.1 & 3.2 & 3.3 & 3.4 & 3.5 & 3.5 & 3.6 & 3.7 & 3.7 \\
\hline .025 & & 2.4 & 2.5 & 2.5 & 2.6 & 2.7 & 2.8 & 2.9 & 3 & 3.1 & 3.2 & 3.3 & 3.4 & 3.5 & 3.5 & 3.6 & 3.7 & 3.7 \\
\hline .05 & & 2.4 & 2.5 & 2.5 & 2.6 & 2.7 & 2.8 & 2.9 & 3 & 3.1 & 3.2 & 3.3 & 3.4 & 3. & 3.4 & 3.5 & 3.6 & 3.7 \\
\hline 0.1 & & 2.4 & 2.5 & 2.5 & 2.6 & 2.7 & 2.8 & 2.9 & 3 & 3.1 & 3.2 & 3.3 & 3.3 & 3.4 & 3.4 & 3.5 & 3.6 & 3.6 \\
\hline
\end{tabular}

Oxygen Percentage at 40 Years as a Function of MCO Power for Various Reactive Surface

Areas, Case of: $8 \mathrm{~kg} \mathrm{Al}(\mathrm{OH}) 3,0.11 \mathrm{~kg} / \mathrm{m}^{\wedge} 2$ water from UO3.2H2O, $200 \mathrm{~g}$ Free H2O, $1.5 \mathrm{~atm}$ abs $\mathrm{He}$ Backfill

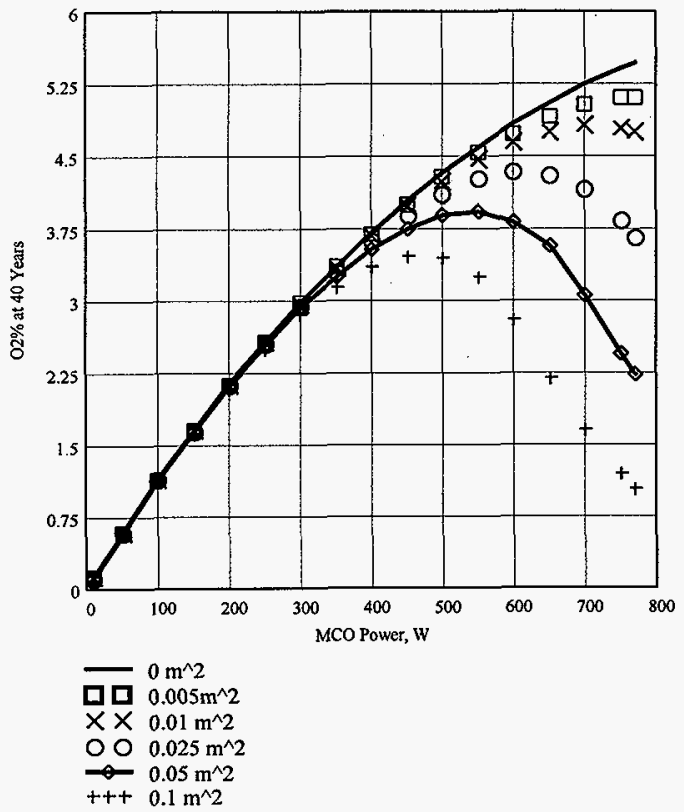




\section{HNF-3035, Rev. 0}

Appendix A

\subsection{RESULTS: $8 \mathrm{AL}(\mathrm{OH}) 3,0.11 \mathrm{~kg} / \mathrm{m}^{\wedge} 2$, RATE MULT=1, $1.5 \mathrm{~atm}$ He BACKFILL}

$\mathrm{m}_{\mathrm{a}}:=0 \quad \mathrm{Al}(\mathrm{OH}) 3$ no mass, grams

$\mathrm{R}=1.0 \quad$ Rate law multiplier

Parametric variation for various power and getter areas:

Power 10 to $770 \mathrm{~W}$, general increments of 50 in the range,

Area up to $5 \mathrm{~m}^{\wedge} 2$ with 0.25 increments below 1.5

$q=0.16 Q_{q}:=50 \cdot q \quad Q_{0}=10 \quad Q_{16}=770$

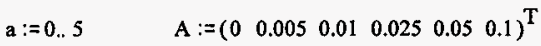

Hydrogen: $\quad \quad n h 2_{q, a}:=\mathrm{fN}_{\mathrm{h} 2}\left(\mathrm{Q}_{\mathrm{q}}, \mathrm{A}_{\mathrm{a}}, \mathrm{m}_{\mathrm{a}}, 40\right)$

Oxygen: $\quad \operatorname{no2}_{\mathrm{q}, \mathrm{a}}:=\mathrm{fN}_{\mathrm{o} 2}\left(\mathrm{Q}_{\mathrm{q}}, \mathrm{A}_{\mathrm{a}}, \mathrm{m}_{\mathrm{a}}, \mathrm{R}, 40\right)$

Helium backfill:

$$
P_{b f}=1.5
$$

$\mathrm{n}_{\mathrm{he}}:=\mathrm{P}_{\mathrm{bf}} 10^{5} \cdot \mathrm{V}_{\mathrm{mco}} \cdot\left(8.314 \mathrm{~T} \mathrm{bf}^{-1} \quad \mathrm{n}_{\mathrm{he}}=30.2716\right.$

Functions for concentration and pressure:

ntot $_{q, a}:=n_{h e}+n_{q, a}+n h 2_{q, a}$

$\mathrm{O} 2 \%_{\mathrm{a}, \mathrm{q}}:=$ no2 $_{\mathrm{q}, \mathrm{a}} \cdot\left(\text { ntot }_{\mathrm{q}, \mathrm{a}}\right)^{-1}$

O2 Mass in grams

$\mathrm{MO} 2_{\mathrm{a}, \mathrm{q}}=\left(\mathrm{if}\left(\mathrm{no} 2_{\mathrm{q}, \mathrm{a}}>0, \mathrm{no}_{\mathrm{q}, \mathrm{a}, \mathrm{a}} 0\right)\right) \cdot 32$

$\mathrm{O} 2 \%_{\mathrm{a}, \mathrm{q}}:=\mathrm{if}\left(\mathrm{O} 2 \%_{\mathrm{a}, \mathrm{q}}>0,100.02 \%_{\mathrm{a}, \mathrm{q}}, 0\right)$

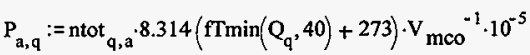

O2 Generation rate at time t, grams/year GIVEN calculation of no2 already (above) as an input:

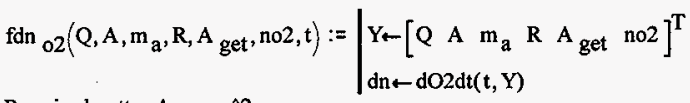

Required getter Area, $\mathrm{m}^{\wedge} 2$ :

$\mathrm{AFE}_{\mathrm{a}, \mathrm{q}}:=\mathrm{fA} \operatorname{get}\left(\frac{\mathrm{MO}_{\mathrm{a}, \mathrm{q}}}{32}, \mathrm{fdn} 02\left(\mathrm{Q}_{\mathrm{q}}, \mathrm{A}_{\mathrm{a}}, \mathrm{m}_{\mathrm{a}}, \mathrm{R}, 0, \frac{\mathrm{MO} 2_{\mathrm{a}, \mathrm{q}}}{32}, 40\right), \mathrm{fTm} \min \left(\mathrm{Q}_{\mathrm{q}}, 40\right)+273\right)$ 
HNF-3035, Rev. 0

Appendix A

Oxygen Percentage at 40 Years as a Function of MCO Power for Various Reactive Surface Areas, Case of: (KE fuel only) no $\mathrm{Al}(\mathrm{OH}) 3,0.11 \mathrm{~kg} / \mathrm{m}^{\wedge} 2$ water from $\mathrm{UO} 3.2 \mathrm{H} 2 \mathrm{O}, 200 \mathrm{~g}$ Free H2O

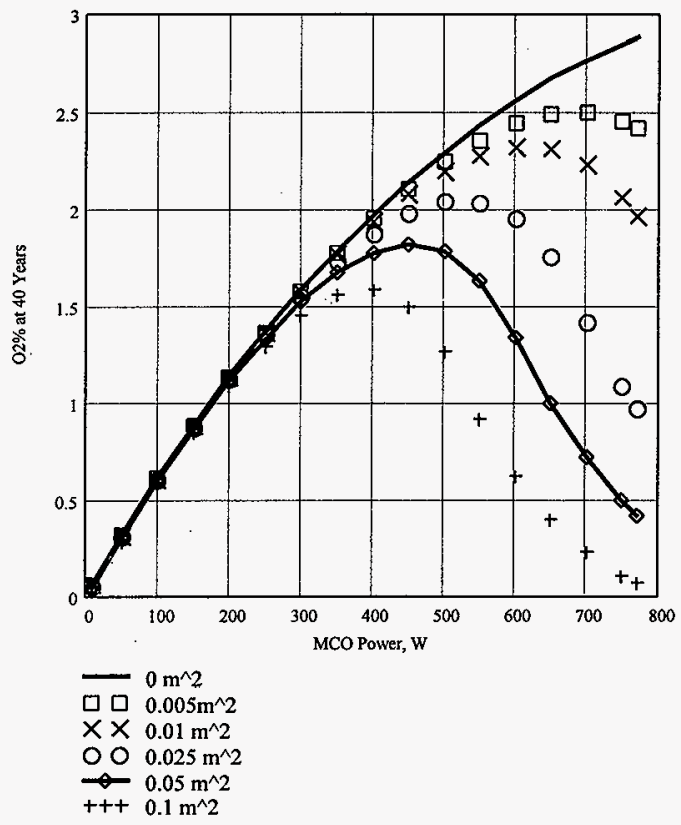

